\title{
Anatomy education during COVID-19; final year medical student's perspective
}

\author{
Humna Iqbal $^{1}$ (D) Masuma Sami $^{1} \cdot$ Naeema Farrah $^{1}$
}

Received: 28 June 2021 / Accepted: 2 August 2021 / Published online: 7 August 2021

(c) The Author(s), under exclusive licence to Springer-Verlag France SAS, part of Springer Nature 2021

\section{To the Editor,}

We read with interest the paper by Singal et al. entitled 'Anatomy education of medical and dental students during COVID-19 pandemic: a reality check' [1].

As a group of final year medical students in the UK, we would like to focus on learning in the dissection room, relating to our experiences. Singal and colleagues reported that many students felt their understanding and confidence was diminished without this learning environment. Reflecting on our own anatomy education, we agree this modality is undoubtedly interesting and often a rite of passage for many medical students; ourselves and our peers can still recall our first experience with the cadaver. Their research briefly mentioned that students missed the interaction with peers and mentors in the dissecting room, a point we believe warrants greater exploration.

In addition to the direct anatomy teaching received during dissections, we believe there is significant impact on the informal and hidden curricula due to COVID-19. These curricula stem from the concept that we learn outside of what we are taught, with interactions and professional culture influencing our education [2]. The dissecting room was one of the earliest environments where our professional identities were cultivated. As pre-clinical students, this was the first interaction we had with clinicians as mentors; peers as colleagues. Dissection is also the first exposure we have to the human body and the first opportunity to practice the duties of the clinician. These include confidentiality, maintaining patient integrity and collaborative teamwork. This learning was fostered under the leadership of an Anatomy Demonstrator, often Foundation Year 3 doctors. As they had completed their clinical training, their teaching would link

Humna Iqbal

humna.iqbal@kcl.ac.uk

1 Faculty of Life Sciences and Medicine, King's College

London, London, UK to clinical application and their own experiences. Through learning anatomy, we were also learning how to be doctors. Notably, our teaching group was one of a minority to be taught by a female demonstrator; she made a concerted effort to encourage participation among the female students and would draw on her experiences of being a woman in surgery-a speciality that continues to be predominantly male-offering advice and encouragement.

Our anatomy education involved live dissections, lectures and online resources. From our experiences, digital anatomy teaching (such as the online workshops, programs and lectures offered by our university) can reasonably replicate dissecting room resources like prosections and potted specimens. In its current form, however, it cannot duplicate the comfortable group mentorship, dynamic teaching and conversation that formed part of our learning in the dissection room.

While previously, a cornerstone of anatomy teaching, many UK medical schools have moved away from mandatory whole-body dissections as a part of the curriculum. With the increasing number of medical students and decline of body donations, dissection-less anatomy teaching may well be the future. As such, we suggest the integration of small-group mentoring alongside the academic anatomy resources that many universities have established. These could span the academic year and be led by anatomy demonstrators, running through teaching, cases or simulations, to mimic learning of the informal curriculum previously learnt through the dissecting room.

\section{References}

1. Singal A, Bansal A, Chaudhary P, Singh H, Patra A (2021) Anatomy education of medical and dental students during COVID19 pandemic: a reality check. Surg Radiol Anat 43(4):515-521. https://doi.org/10.1007/s00276-020-02615-3 
2. Hafferty FW (1998) Beyond curriculum reform: confronting medicine's hidden curriculum. Acad Med 73(4):403-407. https://doi. org/10.1097/00001888-199804000-00013

Publisher's Note Springer Nature remains neutral with regard to jurisdictional claims in published maps and institutional affiliations. 